\title{
El rastreo de la violencia de género y su posterior intervención breve en la mujer asintomática no demostró mejorar resultados en salud
}

\author{
Screening and counselling for violence in asymptomatic women do not show improving health outcomes
}

\section{Objetivos}

Evaluar si la identificación temprana de violencia de género (VG) en mujeres asintomáticas asociada a una intervención psico-conductual realizada por su médico de cabecera produce mejoras en la calidad de vida, salud mental o la planificación de la seguridad de estas.

\section{Diseño, población y lugar}

Ensayo clínico controlado y aleatorizado realizado sobre médicos de familia y sus pacientes mujeres de entre 16 y 50 años, en Victoria, Australia.

\section{Intervención}

Se invitó a participar al 71 y $29 \%$ de los médicos de familia de áreas urbanas y rurales, respectivamente. A su vez, se enviaron encuestas por correo a las pacientes de aquellos médicos que aceptaran participar y que fueron atendidas por estos durante los 12 meses previos. Estas encuestas incluyeron ocho puntos relacionados a salud y estilo de vida e incluyeron como pregunta (valorada mediante una escala de Likert) ¿cuán atemorizada se sintió Ud. por su pareja durante el año previo? A partir de la identificación de mujeres que respondieran afirmativamente sobre este ítem se aleatorizó a sus médicos de cabecera para conformar dos grupos. En el grupo intervención cada médico recibió formación en consejería breve sobre VG (promoviendo la atención centrada en el paciente, escucha activa, entrevista motivacional, preparación para el cambio y acompañamiento) a través de un curso de seis horas de formación no presencial y dos jornadas presenciales interactivas de una hora de duración mediante estrategias simuladas de roll playing. A su vez se invitó a las mujeres afectadas a cargo de cada médico a participar de una a seis sesiones de consejería (según necesidad) durante un periodo de seis meses. En el grupo control los médicos recibieron un
Hegarty K y col. Lancet 2013;382:249-58. paquete de información básica sobre VG y proveyeron el cuidado que consideraran usual para las pacientes que les consultaran por este problema. Todas las mujeres recibieron, junto con las encuestas, un listado de recursos de asistencia ante VG.

\section{Medición de resultados principales}

Se realizó a través de encuestas enviadas por correo postal al momento de la invitación y a los 6 y 12 meses. Los resultados principales evaluados fueron calidad de vida (cuestionario WHO Quality of Life-BREF), salud mental (SF-12), y elaboración de un plan de seguridad. Como resultados secundarios se evaluó ansiedad y depresión.

\section{Resultados}

Se aleatorizaron 25 médicos y sus 137 pacientes (elegibles y que hubieran respondido la encuesta) al grupo intervención; y 27 médicos con sus 135 pacientes al grupo control. No se encontraron diferencias en los resultados principales entre ambos grupos. No se detectaron eventos adversos. Con respecto a los resultados secundarios, se observó una mejoría en la escala de depresión a los 12 meses en el grupo intervención (OR 0,3; IC95\% 0,1 a 0,7).

\section{Conclusiones}

Los resultados de este estudio no apoyan el rastreo por correo de mujeres víctimas de VG. Sin embargo los autores sugieren que los médicos de familia deber recibir entrenamiento para relevar la seguridad de mujeres y niños, y proveer consejería para las mujeres víctimas de abuso, dado que si bien los hallazgos no detectaron mejoras en la calidad de vida, si mostraron reducir la ocurrencia de síntomas depresivos.

Fuente de financiamiento: Australian National Health and Medical Research Council.

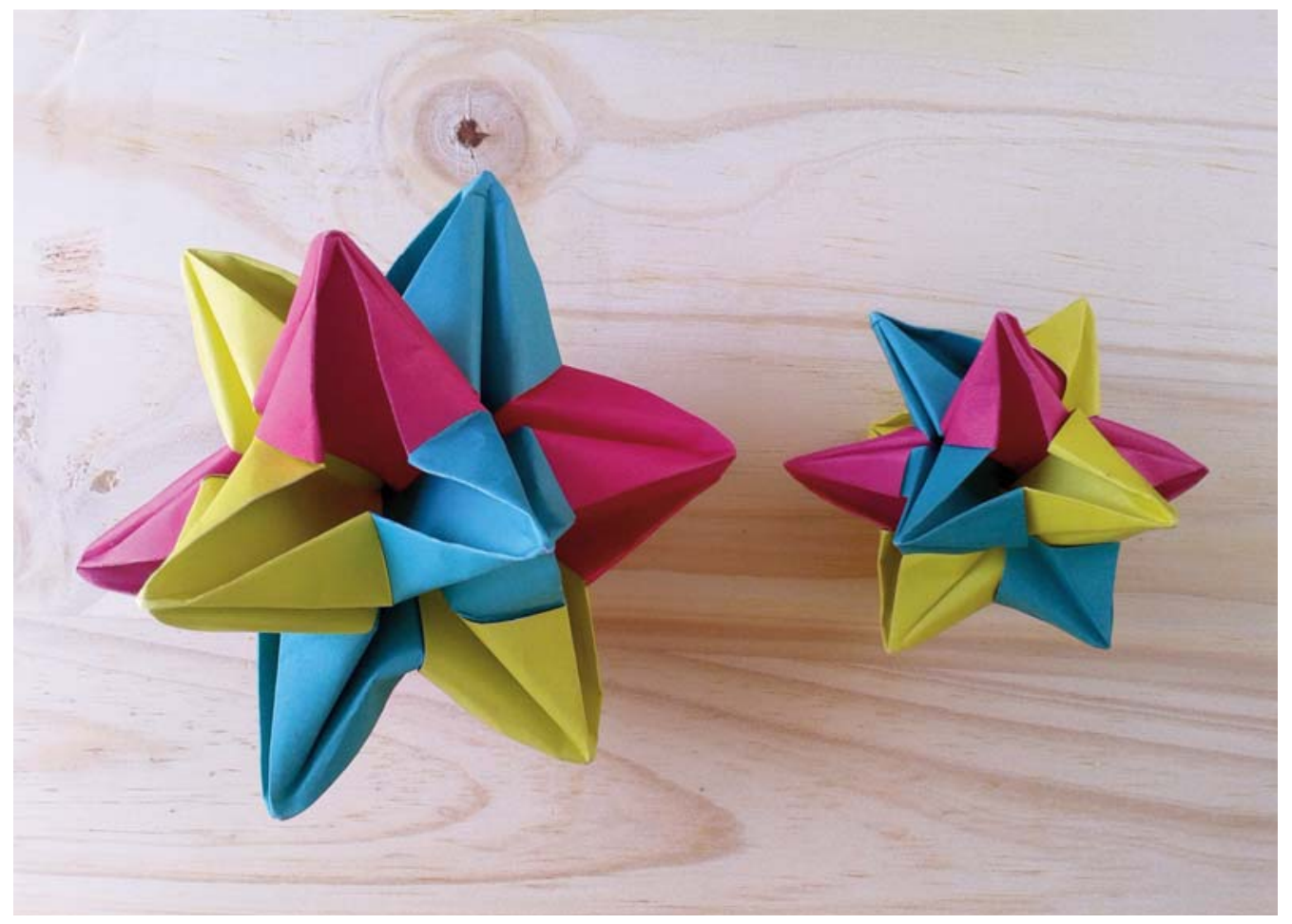

Foto y plegados de Claris Viviano (origami al alma) 


\section{Comentario}

La VG por parte del compañero íntimo es prevalente en todo el mundo y se clasificó como quinta causa de años de vida perdidos debido a la discapacidad en las mujeres ${ }^{1}$. De acuerdo con la Organización de Naciones Unidas (ONU), una de cada tres mujeres va ser golpeada o violada en algún momento de sus vidas. Cuando hablamos de VG no solo nos referimos a un golpe físico, sino a la otra, solapada, no visible que transforma poco a poco la vida de las mujeres. La prevención de la VG y sus consecuencias en la salud son prioridad en la salud pública. Durante muchos años se ha supuesto que los profesionales de la salud tienen un importante rol en identificar mujeres maltratadas y ofrecer una intervención apropiada. Aunque muchas organizaciones han recomendado la identificación de mujeres asintomáticas mediante rastreo, otras, incluidas la OMS, grupos de trabajo de Canadá ${ }^{2}$ y el Programa de Evaluación de Tecnologías del Reino Unido ${ }^{3}$ han opinado lo contrario, concluyendo que no hay suficiente evidencia para sostener tal recomendación. La identificación de mujeres maltratadas haciendo una o más preguntas es factible. Este tipo de intervenciones son atractivas porque son simples y de bajo costo, aún si se ofrece intervenciones de consejería. Este estudio propone una intervención más compleja. Sin embargo los resultados no apoyan su utilidad. La VG es un frecuente factor causal subyacente en las mujeres que se presentan en los servicios de salud, especialmente en los de salud mental. En este contexto consideramos que si sería valioso preguntar sobre VG en aquellas circunstancias en las cuales se presuma que el motivo de consulta este directamente asociado con esta. Abrir la discusión sobre los problemas vinculares, incluyendo la violencia, es necesario para brindar información de cómo esta afecta la salud; de esta forma las mujeres pueden estar mas preparadas para comprender y reconocer que la violencia está relacionado con sus problemas de salud y hacer un cambio en sus vidas. Se debería tener especial atención a los problemas vinculares y a la VG en los servicios que atienden pacientes con HIV y de enfermedades de transmisión sexual, ya que estos afectan la naturaleza del consejo sobre la seguridad en la práctica sexual y el uso de la medicación. Otros estudios que evaluaron intervenciones dirigidas a mujeres atendidas en servicios de control prenatal observaron reducción de la recurrencia de VG y mejoras en la salud de madres y el niños ${ }^{4-5}$. Un trabajo a futuro debería también tener en cuenta el rol del hombre en la VG ya que hay muy poca consideración en cuanto al rastreo de la perpetración de la VG por parte de los hombres y en el ofrecimiento de intervenciones a los hombres para que mejoren sus relaciones y frenen las actitudes violentas.

\section{Conclusiones del comentador}

El estudio comentado evalúa el importante rol de los servicios de salud en la detección y prevención de la violencia doméstica sin llegar aún a encontrar intervenciones que puedan dar respuesta y mejorar la salud de las mujeres. Los médicos de familia, quienes brindamos un seguimiento longitudinal tenemos la oportunidad de detectar e intervenir en este problema y deberemos seguir explorando nuestro rol en la detección de mujeres que padecen maltrato. Sin embargo es también necesario tener una visión integral del tema para poder hacer un cambio profundo y comprender que este es un tema de la sociedad en su conjunto, donde la educación debe cumplir un papel fundamental, incluso desde los ciclos iniciales, implantando la problemática de género en general y de la violencia contra la mujer en particular.

Adriana Goldman [ Servicio de Medicina Familiar y Comunitaria del Hospital Italiano de Buenos Aires. adriana.goldman@ hospitalitaliano.org.ar ]

Goldman A. El rastreo de la violencia de género y su posterior intervención breve en la mujer asintomática no demostró mejorar resultados en salud. Evid Actual Práct Ambul; 2015 18(3): 76-77. Jul-Sep. Comentado de: Hegarty K, O’Doherty L, Taft A, y col. Screening and counselling in the primary care setting for women who have experienced intimate partner violence (WEAVE): a cluster randomised controlled trial. Lancet 2013;382:249-58. PMID: 23598181.

\section{Referencias:}

1. Global Burden of Disease Study 2013 Collaborators. Global, regional, and national incidence, prevalence, and years lived with disability for 301 acute and chronic diseases and injuries in 188 countries, 1990-2013: a systematic analysis for the Global Burden of Disease Study 2013. Lancet. 2015 Jun 7. pii:S0140-6736(15)60692-4.

2. Wathen CN, MadMillan HL Prevention of violence against women: recommendation statement from the Canadian Task Force on Preventive Health Care. CMAJ 2003;169:582-84.

3. Feder G, Ramsay J, Dunne D, y col. How far does screening women for domestic (partner) violence in diff erent health-care settings meet criteria for a screening programme? Systematic reviews of nine UK National Screening Committee criteria. Health Technol Assess 2009;13:1-347.

4. Kiely M, El-Mohandes AA, El-Khorazaty MN, y col. An integrated intervention to reduce intimate partner violence in pregnancy: a randomized controlled trial. Obstet Gynecol 2010;115: 273-83.

5. Tiwari A, Leung WC, Leung TW, y col. A randomized controlled trial of empowerment training for Chinese abused pregnant women in Hong Kong. BJOG. 2005 Sep;112(9):1249-56. 